\title{
Noncommutative Geometry: Fuzzy Spaces, the Groenewold-Moyal Plane
}

\author{
Aiyalam P. BALACHANDRAN and Babar Ahmed QURESHI \\ Department of Physics, Syracuse University, Syracuse, NY, USA \\ E-mail: bal@phy.syr.edu,bqureshi@phy.syr.edu
}

Received September 22, 2006, in final form December 14, 2006; Published online December 29, 2006

Original article is available at http://www.emis.de/journals/SIGMA/2006/Paper094/

\begin{abstract}
In this talk, we review the basics concepts of fuzzy physics and quantum field theory on the Groenewold-Moyal Plane as examples of noncommutative spaces in physics. We introduce the basic ideas, and discuss some important results in these fields. At the end we outline some recent developments in the field.
\end{abstract}

Key words: noncommutative geometry; quantum algebra; quantum field theory

2000 Mathematics Subject Classification: 81R60; 46L65

\section{Introduction}

Noncommutative geometry is a branch of mathematics due to Gel'fand, Naimark, Connes, Rieffel and many others $[1,2,3]$. Physicists in a very short time adopted it and nowadays use this phrase whenever spacetime algebra is noncommutative.

There are two such particularly active fields in physics at present

1. Fuzzy Physics,

2. Quantum Field Theory (QFT) on the Groenewald-Moyal Plane.

Item 1 is evolving into a tool to regulate QFT's, and for numerical work. It is an alternative to lattice methods. Item 2 is more a probe of Planck-scale physics. This introductory talk will discuss both items 1 and 2 .

\section{History}

The Groenewold-Moyal (G-M) plane is associated with noncommutative spacetime coordinates:

$$
\left[x_{\mu}, x_{\nu}\right]=i \theta_{\mu \nu}
$$

It is an example where spacetime coordinates do not commute.

The idea that spatial coordinates may not commute first occurs in a letter from Heisenberg to Peierls $[4,5]$. Heisenberg suggested that an uncertainty principle such as

$$
\Delta x_{\mu} \Delta x_{\nu} \geq \frac{1}{2}\left|\theta_{\mu \nu}\right|, \quad \theta_{\mu \nu}=\mathrm{const}
$$

${ }^{\star}$ This paper is a contribution to the Proceedings of the O'Raifeartaigh Symposium on Non-Perturbative and Symmetry Methods in Field Theory (June 22-24, 2006, Budapest, Hungary). The full collection is available at http://www.emis.de/journals/SIGMA/LOR2006.html 
can provide a short distance cut-off and regulate quantum field theories (qft's). In this letter, he apparently complains about his lack of mathematical skills to study this possibility. Peierls communicated this idea to Pauli, Pauli to Oppenheimer and finally Oppenheimer to Snyder. Snyder wrote the first paper on the subject [6]. This was followed by a paper of Yang [7].

In mid-90's, Doplicher, Fredenhagen and Roberts [8, 9] systematically constructed unitary quantum field theories on the G-M plane and its generalizations, even with time-space noncommutativity.

Later string physics encountered these structures.

\section{What is noncommutative geometry}

According to Connes $[1,2,3]$, noncommutative geometry is a spectral triple,

$$
(\mathcal{A}, D, \mathcal{H})
$$

where $\mathcal{A}=$ a $C^{*}$-algebra, possibly noncommutative, $D=$ a Dirac operator, $\mathcal{H}=$ a Hilbert space on which they are represented.

If $\mathcal{A}$ is a commutative $C^{*}$-algebra, we can recover a Hausdorff topological space on which $\mathcal{A}$ are functions,using theorems of Gel'fand and Naimark. But that is not possible if $\mathcal{A}$ is not commutative. But it is still possible to formulate qft's using the spectral triple. A class of examples of noncommutative geometry with $\mathcal{A}$ noncommutative is due to Connes and Landi [10].

If some of the strict axioms are not enforced then the examples include $S U(2)_{q}$, fuzzy spaces, the G-M plane, and many more.

The introduction of noncommutative geometry has introduced a conceptual revolution. Manifolds are being replaced by their "duals", algebras, and these duals are being "quantized", much as in the passage from classical to quantum mechanics.

\section{$4 \quad$ Fuzzy physics}

In what follows, we sketch the contents of "fuzzy physics". Reference [11] contains a detailed survey. For pioneering work on fuzzy physics, see $[12,13,14]$.

\subsection{What is fuzzy physics [11]}

We explain the basic ideas of fuzzy physics by a two-dimensional example: $S_{F}^{2}$.

Consider the two-sphere $S^{2}$. We quantize it to regularize by introducing a short distance cut-off. For example in classical mechanics, the number of states in a phase space volume

$$
\Delta V=d^{3} p d^{3} q
$$

is infinite. But we know since Planck and Bose that on quantization, it becomes

$$
\frac{\Delta V}{h^{3}}=\text { finite. }
$$

This is the idea behind fuzzy regularization.

In detail, this regularization works as follows on $S^{2}$. We have

$$
S^{2}=\left[\vec{x} \in \mathbb{R}^{3}: \vec{x} \cdot \vec{x}=r^{2}\right] .
$$

Now consider angular momentum $L_{i}$ :

$$
\left[L_{i}, L_{j}\right]=i \epsilon_{i j k} L_{k}, \quad \vec{L}^{2}=l(l+1) .
$$


Set

$$
\begin{aligned}
& \hat{x}_{i}=r \frac{L_{i}}{\sqrt{l(l+1)}} \Rightarrow \\
& \hat{x} \cdot \hat{x}=r^{2}, \quad\left[\hat{x}_{i}, \hat{x}_{j}\right]=\frac{r}{\sqrt{l(l+1)}} i \epsilon_{i j k} \hat{x}_{k},
\end{aligned}
$$

where $\hat{x}_{i} \in \operatorname{Mat}_{2 l+1} \equiv$ space of $(2 l+1) \times(2 l+1)$ matrices. As $l \rightarrow \infty$, they become commutative. They give the fuzzy sphere $S_{F}^{2}$ of radius $r$ and dimensions $2 l+1$.

\subsection{Why is this space fuzzy}

As $\hat{x}_{i}, \hat{x}_{j}(i \neq j)$ do not commute, we cannot sharply localize $\hat{x}_{i}$. Roughly in a volume $4 \pi r^{2}$ there are $(2 l+1)$ states.

\subsection{Field theory on fuzzy sphere}

A scalar field on fuzzy sphere is defined as a polynomial in $\hat{x}_{i}$, i.e.,

A scalar field $\Phi=$ A polynomial in $\hat{x}_{i}=\mathrm{A}(2 l+1)-$ dimensional matrix.

Differentiation is given by infinitesimal rotation:

$$
\mathcal{L}_{i} \Phi=\left[L_{i}, \Phi\right]
$$

A simple rotationally invariant scalar field action is given by

$$
S(\Phi)=\mu \operatorname{Tr}\left[L_{i}, \Phi\right]^{\dagger}\left[L_{i}, \Phi\right]+\frac{m}{2} \operatorname{Tr}\left(\Phi^{\dagger} \Phi\right)+\lambda \operatorname{Tr}\left(\Phi^{\dagger} \Phi\right)^{2}
$$

Simulations have been performed $[15,16]$ on the partition function $\mathcal{Z}=\int d \Phi e^{-S(\Phi)}$ of this model and the major findings include the following:

- Continuum limit exists.

- If

$$
\Phi=\sum c_{l m} \hat{Y}_{l m}, \quad \hat{Y}_{l m}=\text { spherical tensor }
$$

then there are three phases:

1. Disordered : $\left\langle\sum\left|c_{l m}\right|^{2}\right\rangle=0$.

2. Uniform ordered: $\left\langle\left|c_{00}\right|^{2}\right\rangle \neq 0,\left\langle\left|c_{l m}\right|^{2}\right\rangle=0$ for $l \neq 0$.

3. Non-uniform ordered: $\left\langle\left|c_{1 m}\right|^{2}\right\rangle \neq 0,\left\langle\mid c_{l m}^{2}\right\rangle=0$ for $l \neq 1$.

The last one is an analogue of the Gupser-Sondhi phase [17, 18, 19].

\subsubsection{Dirac operator}

$S_{F}^{2}$ has a Dirac operator including instantons and with no fermion doubling $[20,21,22,23,24$, $25,26]$.

Also $S_{F}^{2}$ can nicely describe topological features. Hence it seems better suited for preserving symmetries than lattice approximations. 


\subsubsection{Supersymmetry}

If we replace $S U(2)$ by $O S p(2,1)$, the fuzzy sphere becomes the $N=1$ supersymmetric fuzzy sphere and can be used to simulate supersymmetry [20, 21, 27, 28, 29, 30, 31, 32]. Simulations in this regard are already starting.

\subsubsection{Strings [33]}

If $N D$-branes are close, the transverse coordinates $\Phi_{i}$ become $N \times N$ matrices with the action given by

$$
\mathrm{S}=\lambda \operatorname{Tr}\left[\Phi_{i}, \Phi_{j}\right]^{\dagger}\left[\Phi_{i}, \Phi_{j}\right]+i f_{i j k} \Phi_{i} \Phi_{j} \Phi_{k},
$$

where $f_{i j k}$ are totally antisymmetric.

The equations of motion

$$
\left[\Phi_{i}, \Phi_{j}\right] \cong i f_{i j k} \Phi_{k}
$$

give solutions when $f_{i j k}$ are structure constants of a simple compact Lie group. Thus we can have

$$
\Phi_{i}=c L_{i}, \quad f_{i j k}=c \epsilon_{i j k}, \quad c=\text { const }, \quad L_{i}=\text { angular momentum operators } .
$$

If $L_{i}$ form an irreducible set, then we have

$$
\vec{L} \cdot \vec{L}=l(l+1), \quad(2 l+1)=N,
$$

and we have one fuzzy sphere. Or we can have a direct sum of irreducible representations:

$$
L_{i}=\oplus L_{i}^{l_{k}}, \quad \vec{L}^{l_{k}} \cdot \vec{L}^{l_{k}}=l_{k}\left(l_{k}+1\right), \quad \sum\left(2 l_{k}+1\right)=N
$$

Then we have many fuzzy spheres.

Stability analysis of these solutions including numerical studies has been done by many groups.

\section{The G-M Plane}

\subsection{Quantum gravity and spacetime noncommutativity: heuristics}

The following arguments were described by Doplicher, Fredenhagen and Robert in their work in support of the necessity of noncommutative spacetime at Planck scale.

\subsubsection{Space-space noncommutativity}

In order to probe physics at the Planck scale $L$, the Compton wavelength $\frac{\hbar}{M c}$ of the probe must fulfill

$$
\frac{\hbar}{M c} \leq L \quad \text { or } \quad M \geq \frac{\hbar}{L c} \simeq \text { Planck mass }
$$

Such high mass in the small volume $L^{3}$ will strongly affect gravity and can cause black holes to form. This suggests a fundamental length limiting spatial localization. 


\subsubsection{Time-space noncommutativity}

Similar arguments can be made about time localization. Observation of very short time scales requires very high energies. They can produce black holes and black hole horizons will then limit spatial resolution suggesting

$$
\Delta t \Delta|\vec{x}| \geq L^{2}, \quad L=\text { a fundamental length. }
$$

The G-M plane models above spacetime uncertainties.

\subsection{What is the G-M plane}

The Groenewald-Moyal plane $\mathcal{A}_{\theta}\left(\mathbb{R}^{d+1}\right)$ consists of functions $\alpha, \beta, \ldots$ on $\mathbb{R}^{d+1}$ with the $*$-product

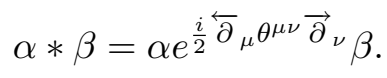

For spacetime coordinates, this implies,

$$
\left[x_{\mu}, x_{\nu}\right]_{*}=x_{\mu} * x_{\nu}-x_{\nu} * x_{\mu}=i \theta_{\mu \nu}
$$

Conversely these coordinate commutators imply the general $*$-product up to certain equivalencies.

The G-M plane also emerges in quantum Hall effect and string physics.

\subsection{How the G-M plane emerges from quantum Hall effect and strings}

\subsubsection{Quantum Hall effect (the Landau problem) [34]}

Consider an electron in 1-2 plane and an external magnetic field $\vec{B}=(0,0, B)$ perpendicular to the plane. Then the Lagrangian for the system is

$$
L=\frac{1}{2} m \dot{x}_{a}^{2}+e \dot{x}_{a}^{2} A_{a}
$$

where

$$
A_{a}=-\frac{B}{2} \epsilon_{a b} x^{b}, \quad a, b=1,2,
$$

is the electromagnetic potential and $x_{a}$ are the coordinates of the electron.

Now if $e B \rightarrow \infty$, then

$$
L \sim \frac{e B}{2}\left(\dot{x}_{1} x_{2}-\dot{x}_{2} x_{1}\right)
$$

This means that on quantization we will have

$$
\left[\hat{x}_{a}, \hat{x}_{b}\right]=\frac{i}{e B} \epsilon_{a b}
$$

which defines a G-M plane. 


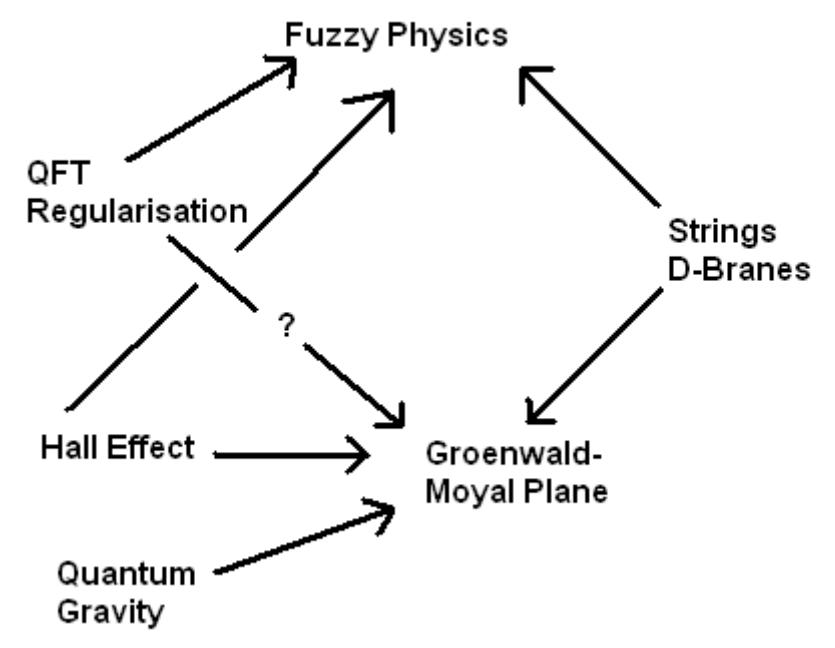

Figure 1. The tangled web: emergence of noncommutative spaces from different fields.

\subsubsection{Strings [35]}

Consider open strings ending on $D p$-Branes. If there is a background two-form Neveu-Schwarz field given by the constants $B_{i j}=-B_{j i}$, then the action is given by

$$
S_{\Sigma}=\frac{1}{4 \pi \alpha^{\prime}} \int_{\Sigma}\left[g_{i j} \partial_{a} x^{i} \partial_{a} x^{j}-2 \pi \alpha^{\prime} B_{i j} \partial_{a} x^{i} \partial_{b} x^{j} \epsilon^{a b}+\text { spinor terms }\right] d \sigma d t .
$$

As $B \rightarrow \infty$ or equivalently $g_{i j} \rightarrow 0$,

$$
\begin{aligned}
& S_{\Sigma}=-\frac{2 \pi e}{4 \pi} \int_{\Sigma} B_{i j} d x^{i} \wedge d x^{j}=\left[\int_{\partial \Sigma^{0}}-\int_{\partial \Sigma^{1}}\right] e B_{i j} x^{i} \frac{d x^{j}}{d t} \\
& \Rightarrow \quad e\left[B_{i j} \hat{x}^{j}, \hat{x}^{k}\right]=i \delta_{i k} \quad \text { or } \quad\left[\hat{x}_{j}, \hat{x}_{k}\right]=\frac{i}{e}\left(B^{-1}\right)_{j k}
\end{aligned}
$$

which is just a G-M plane.

Fig. 1 indicates different sources wherefrom fuzzy physics and the G-M plane emerge. The question mark is to indicate that the G-M plane may not regularize qft's.

\subsection{Prehistory (before $2004 / 2005$ )}

Until 2004/2005, much work was done on

- QFT's on the G-M plane and its renormalization theory, uncovering the phenomenon of $\mathrm{UV} / \mathrm{IR}$ mixing [36].

- Phenomenology, including the study of the effects of noncommutativity on Lorentz invariance violation (from $\theta_{\mu \nu}$ in $\left.\left[x_{\mu}, x_{\nu}\right]_{*}=i \theta_{\mu \nu}\right), \mathrm{C}, \mathrm{CP}$ and CPT.

\subsection{Modern era}

In 2004/2005, Chaichian et al. [37, 38] and Aschieri et al. [39, 40] applied the Drinfel'd twist [41] which restores full diffeomorphism invariance (with a twist in the "coproduct") despite the presence of constants $\theta_{\mu \nu}$ in $\left[\hat{x}_{\mu}, \hat{x}_{\nu}\right]=i \theta_{\mu \nu}$. This twist also twists statistics $[42,43]^{1}$.

Much of this was known to Majid [47], Oeckl [48], Fiore and Schupp [49, 50, 51] and Watts [52, 53]. So the Drinfel'd twist twists both

\footnotetext{
${ }^{1}$ There are claims to the contrary, see $[44,45,46]$ for the debate.
} 
1. action of diffeomorphisms, and

2. exchange statistics.

This brings into question much of the prehistory-analysis. Examples include the following new results:

1. The Pauli principle can be violated on the G-M plane.

2. (Twisted) Lorentz invariance need not be violated even if $\theta_{\mu \nu} \neq 0$.

3. There need be no ultraviolet-infrared (UV-IR) mixing in the absence of gauge fields [54].

There is also a striking, clean separation of matter from gauge fields due to the Drinfel'd twist [55], (in the sense that they have to be treated differently) reminiscent of the distinction between particles and waves in the classical theory.

Literature should be consulted for details of these developments.

\section{Acknowledgments}

This work was supported by DOE under grant number DE-FG02-85ER40231.

[1] Connes A., Noncommutative geometry, San Diego, CA, Academic Press, 1994.

[2] Varilly J.C., Figueroa H., Gracia-Bondia J.M., Elements of noncommutative geometry, Boston, Birkhauser, 2000.

[3] Landi G., Introduction to noncommutative spaces and their geometries, New York, Springer Verlag, 1997.

[4] Jackiw R., Physical instances of noncommuting coordinates, Nuclear Phys. Proc. Suppl., 2002, V.108, 30-36, hep-th/0110057.

[5] Pauli W., Letter of Heisenberg to Peirels (1930), in Wolfgang Pauli, Scientific Correspondence, Vol. II, Springer Verlag, 1985, 15-15.

[6] Snyder H., Quantized space-time, Phys. Rev., 1947, V.71, 38-41.

[7] Yang C.N., On quantized space-time, Phys. Rev., 1947, V.72, 874-874.

[8] Doplicher S., Fredenhagen K., Roberts J., Space-time quantization induced by classical gravity, Phys. Lett. B, 1994, V.331, 39-44.

[9] Doplicher S., Fredenhagen K., Roberts J., The quantum structure of space-time at the Planck scale and quantum fields, Comm. Math. Phys., 1995, V.172, 187-220, hep-th/0303037.

[10] Connes A., Landi G., Noncommutative manifolds: the instanton algebra and isospectral deformations, Comm. Math. Phys., 2001, V.221, 141-159, math.QA/0011194.

[11] Balachandran A.P., Kurkcuoglu S., Vaidya S., Lectures on fuzzy and fuzzy susy physics, World Scientific, to appear, hep-th/0511114.

[12] Hoppe J., Quantum theory of a massless relativistic surface and a two dimensional bound state problem, PhD Thesis, MIT, 1982.

[13] Madore J., The fuzzy sphere, Classical Quantum Gravity, 1992, V.9, 69-88.

[14] Madore J., An introduction to non-commutative differential geometry and its physical applications, Cambridge, Cambridge University Press, 1995.

[15] Martin X., A matrix phase for the $\phi^{4}$ scalar field on the fuzzy sphere, JHEP, 2004, N 4, Paper 077,15 pages, hep-th/0402230.

[16] Flores F.G., O'Connor D., Martin X., Simulating the scalar field on the fuzzy sphere, in Proceedings for the XXIIIrd International Symposium on Lattice Field Theory, PoSLat2005, 2006, 262, 6 pages, hep-lat/0601012.

[17] Gubser S.S., Sondhi S.L., Phase structure of non-commutative scalar field theories, Nuclear Phys. B, 2001, V.605, 395-424, hep-th/0006119.

[18] Ambjorn J., Catterall S., Stripes from (noncommutative) stars, Phys. Lett. B, 2002, V.549, 253-259, hep-lat/0209106.

[19] Medina J., Bietenholz W., Hofheinz F., O'Connor D., Field theory simulations on a fuzzy sphere - an alternative to the lattice, PoSLAT2005, 2006, 263, 6 pages, hep-lat/0509162. 
[20] Grosse H., Klimčik C., Prešnajder P., Field theory on a supersymmetric lattice, Comm. Math. Phys., 1997, V.185, 155-175, hep-th/9507074.

[21] Grosse H., Klimčik C., Prešnajder P., $N=2$ superalgebra and non-commutative geometry, hep-th/9603071.

[22] Balachandran A.P., Immirzi G., The fuzzy Ginsparg-Wilson algebra: a solution of the fermion doubling problem, Phys. Rev. D, 2003, V.68, 065023, 7 pages, hep-th/0301242.

[23] Carow-Watamura U., Watamura S., Chirality and Dirac operator on noncommutative sphere, Comm. Math. Phys., 1997, V.183, 365-382, hep-th/9605003.

[24] Baez S., Balachandran A.P., Ydri B., Vaidya S., Monopoles and solitons in fuzzy physics, Comm. Math. Phys., 2000, V.208, 787-798, hep-th/9811169.

[25] Balachandran A.P., Govindarajan T.R., Ydri B., The fermion doubling problem and noncommutative geometry, Modern Phys. Lett. A, 2000, V.15, 1279-1286, hep-th/9911087.

[26] Balachandran A.P., Govindarajan T.R., Ydri B., Fermion doubling problem and noncommutative geometry II, hep-th/0006216.

[27] Grosse H., Reiter G., The fuzzy supersphere, J. Geom. Phys., 1998, V.28, 349-383, math-ph/9804013.

[28] Klimčik C., A nonperturbative regularization of the supersymmetric Schwinger model, Comm. Math. Phys., 1999, V.206, 567-586, hep-th/9903112.

[29] Klimčik C., An extended fuzzy supersphere and twisted chiral superfields, Comm. Math. Phys., 1999, V.206, 587-601, hep-th/9903202.

[30] Balachandran A.P., Kurkcuoglu S., Rojas E., The star product on the fuzzy supersphere, JHEP, 2002, N 7, Paper 056, 21 pages, hep-th/0204170.

[31] Balachandran A.P., Pinzul A., Qureshi B., SUSY anomalies break $N=2$ to $N=1$ : the supersphere and the fuzzy supersphere, JHEP, 2005, N 12, Paper 002, 14 pages, hep-th/0506037.

[32] Kurkcuoglu S., Non-linear sigma model on the fuzzy supersphere, JHEP, 2004, N 3, Paper 062, 11 pages, hep-th/0311031.

[33] Szabo R.J., D-branes in noncommutative field theory, hep-th/0512054.

[34] Ezawa Z.F., Tsitsishvili G., Hasebe K., Noncommutative geometry, extended $W_{\infty}$ algebra and Grassmannian solitons in multicomponent quantum Hall systems, Phys. Rev. B, 2003, V.67, 125314, 15 pages, hep-th/0209198.

[35] Seiberg N., Witten E., String theory and noncommutative geometry, JHEP, 1999, N 9, Paper 032, 100 pages, hep-th/9908142.

[36] Minwala S., van Raamsdonk M., Seiberg N., Noncommutative perturbative dynamics, JHEP, 2000, N 2, Paper 020, 33 pages, hep-th/9912072.

[37] Chaichian M., Kulish P.P., Nishijima K., Tureanu A., On a Lorentz-invariant interpretation of noncommutative space-time and its implications on noncommutative QFT, Phys. Lett. B, 2004, V.604, 98-102, hep-th/0408069.

[38] Chaichian M., Presnajder P., Tureanu A., New concept of relativistic invariance in NC space-time: twisted Poincaré symmetry and its implications, Phys. Rev. Lett., 2005, V.94, 151602, 15 pages, hep-th/0409096.

[39] Dimitrijevic M., Wess J., Deformed bialgebra of diffeomorphisms, hep-th/0411224.

[40] Aschieri P., Blohmann C., Dimitrijevic M., Meyer F., Schupp P., Wess J., A gravity theory on noncommutative spaces, Classical Quantum Gravity, 2005, V.22, 3511-3532, hep-th/0504183.

[41] Drinfel'd V.G., Quasi Hopf algebras, Leningrad Math. J., 1990, V.1, 1419-1457.

[42] Balachandran A.P., Mangano G., Pinzul A., Vaidya S., Spin and statistics on the Groenewold-Moyal plane: Pauli-Forbidden levels and transitions, Internat. J. Modern Phys. A, 2006, V.21, 3111-3126, hep-th/0508002.

[43] Qureshi B.A., Twisted supersymmetry, fermion-boson mixing and removal of UV-IR mixing, hep-th/0602040.

[44] Tureanu A., Twist and spin-statistics relation in noncommutative quantum field theory, Phys. Lett. B, 2006, V.638, 296-301, hep-th/0603219.

[45] Zahn J., Remarks on twisted noncommutative quantum field theory, Phys. Rev. D, 2006, V.73, 105005, 13 pages, hep-th/0603231.

[46] Balachandran A.P., Govindarajan T.R., Mangano G., Pinzul A., Qureshi B.A., Vaidya S., Statistics and UV-IR mixing with twisted Poincaré invariance, hep-th/0608179.

[47] Majid S., Foundations of quantum group theory, Cambridge University Press, 1995. 
[48] Oeckl R., Untwisting noncommutative $R^{d}$ and the equivalence of quantum field theories, Nuclear Phys. B, 2000, V.581, 559-574, hep-th/0003018.

[49] Fiore G., Schupp P., Statistics and quantum group symmetries, in Quantum Groups and Quantum Spaces, Banach Centre Publications, Vol. 40, Warszawa, Institute of Mathematics, Polish Academy of Sciences, 1997, 369-377.

[50] Fiore G., Deforming maps and Lie group covariant creation and annihilation operators, J. Math. Phys., 1998, V.39, 3437-3452, q-alg/9610005.

[51] Fiore G., Schupp P., Identical particles and quantum symmetries, Nuclear Phys. B, 1996, V.470, 211-235, hep-th/9508047.

[52] Watts P., Noncommutative string theory, the R-matrix and Hopf algebras, Phys. Lett. B, 2000, V.474, 295-302, hep-th/9911026.

[53] Watts P., Derivatives and the role of the Drinfel'd twist in noncommutative string theory, hep-th/0003234.

[54] Balachandran A.P., Pinzul A., Qureshi B.A., UV-IR mixing in non-commutative plane, Phys. Lett. B, 2006, V.634, 434-436, hep-th/0508151.

[55] Balachandran A.P., Pinzul A., Qureshi B.A., Vaidya S., Poincaré invariant gauge and gravity theories on the Groenewold-Moyal plane, hep-th/0608138. 